\title{
Wave-activity conservation laws for the three-dimensional anelastic and Boussinesq equations with a horizontally homogeneous background flow
}

Article

Published Version

Shaw, T. A. and Shepherd, T. G. (2008) Wave-activity conservation laws for the three-dimensional anelastic and Boussinesq equations with a horizontally homogeneous background flow. Journal Of Fluid Mechanics, 594. pp. 493506. ISSN 0022-1120 doi: https://doi.org/10.1017/S0022112007009160 Available at https://centaur.reading.ac.uk/31775/

It is advisable to refer to the publisher's version if you intend to cite from the work. See Guidance on citing.

To link to this article DOI: http://dx.doi.org/10.1017/S0022112007009160

Publisher: Cambridge University Press

All outputs in CentAUR are protected by Intellectual Property Rights law, including copyright law. Copyright and IPR is retained by the creators or other copyright holders. Terms and conditions for use of this material are defined in the End User Agreement. 


\section{www.reading.ac.uk/centaur}

\section{CentAUR}

Central Archive at the University of Reading

Reading's research outputs online 


\title{
Wave-activity conservation laws for the three-dimensional anelastic and Boussinesq equations with a horizontally homogeneous background flow
}

\author{
TIFFANY A. SHAW AND THEODORE G. SHEPHERD
}

Department of Physics, University of Toronto, Toronto, ON, Canada, M5S 1A7

(Received 23 July 2007 and in revised form 17 September 2007)

Wave-activity conservation laws are key to understanding wave propagation in inhomogeneous environments. Their most general formulation follows from the Hamiltonian structure of geophysical fluid dynamics. For large-scale atmospheric dynamics, the Eliassen-Palm wave activity is a well-known example and is central to theoretical analysis. On the mesoscale, while such conservation laws have been worked out in two dimensions, their application to a horizontally homogeneous background flow in three dimensions fails because of a degeneracy created by the absence of a background potential vorticity gradient. Earlier three-dimensional results based on linear WKB theory considered only Doppler-shifted gravity waves, not waves in a stratified shear flow. Consideration of a background flow depending only on altitude is motivated by the parameterization of subgrid-scales in climate models where there is an imposed separation of horizontal length and time scales, but vertical coupling within each column. Here we show how this degeneracy can be overcome and wave-activity conservation laws derived for three-dimensional disturbances to a horizontally homogeneous background flow. Explicit expressions for pseudoenergy and pseudomomentum in the anelastic and Boussinesq models are derived, and it is shown how the previously derived relations for the two-dimensional problem can be treated as a limiting case of the three-dimensional problem. The results also generalize earlier three-dimensional results in that there is no slowly varying WKB-type requirement on the background flow, and the results are extendable to finite amplitude. The relationship $A^{\mathscr{E}}=c A^{\mathscr{P}}$ between pseudoenergy $A^{\mathscr{E}}$ and pseudomomentum $A^{\mathscr{P}}$, where $c$ is the horizontal phase speed in the direction of symmetry associated with $A^{\mathscr{P}}$, has important applications to gravity-wave parameterization and provides a generalized statement of the first Eliassen-Palm theorem.

\section{Introduction}

It is common in fluid dynamical applications to consider the fluid state as a disturbance to some specified background state. In such cases wave-activity conservation laws play a central role. In the case of the large-scale circulation of the middle atmosphere, the Eliassen-Palm wave activity has been crucial to theoretical analysis (Andrews, Holton \& Leovy 1987). In the case of the mesoscale, whose interaction with the large scales is represented in climate models through subgridscale parameterizations, one generally considers three-dimensional disturbances to a horizontally homogeneous but vertically dependent background flow. 
Previous studies have treated three-dimensional Boussinesq disturbances to a planeparallel shear flow in the context of gravity-wave propagation (Eliassen \& Palm 1961; Bretherton 1966; Hines \& Reddy 1967). However, all those studies ignored curvature in the background flow and were basically treating Doppler-shifted gravity waves (in a varying stratification), rather than waves in a stratified shear flow. Furthermore those theories are linear, and limited to WKB conditions in the vertical. What one desires for practical applications are finite-amplitude wave-activity conservation laws that do not require WKB assumptions. By a wave-activity conservation law, we mean a relation of the form

$$
\frac{\partial A}{\partial t}=-\nabla \cdot \boldsymbol{F}
$$

for the conservative dynamics, where $A$ is the density of the wave activity and $\boldsymbol{F}$ its flux, both being quadratic in disturbance amplitude in the small-amplitude limit. (In the presence of forcing or dissipation, the equation would also include a term that was not the divergence of a flux.)

Wave-activity conservation laws can be generally derived within a Hamiltonian framework (Shepherd 1990, 2003). This framework should, in principle, allow one to consider general disturbances to a stratified shear flow, without assuming WKB conditions in the vertical, and be extendable to finite amplitude. However the application of this approach to the case of three-dimensional disturbances to a horizontally homogeneous background flow is hindered by the lack of a background potential vorticity gradient. (The two-dimensional results of Scinocca \& Shepherd (1992) require a Casimir invariant that is particular to the two-dimensional geometry.) Abarbanel et al. (1986) showed how such a degeneracy can be overcome, for the case of the three-dimensional Boussinesq equations, in their derivation of a nonlinear stability criterion for stratified plane-parallel flows. Here we apply the technique of Abarbanel et al. (1986) to the three-dimensional anelastic and Boussinesq equations with a horizontally homogeneous background flow, and derive wave-activity conservation laws associated with energy and horizontal momentum.

We begin $(\S 2)$ by introducing the three-dimensional anelastic and Boussinesq systems, and present the Hamiltonian structure of the two sets of equations including the structure of the Casimir invariants. The wave-activity conservation laws for disturbances to a plane-parallel background flow are derived in $\S 3$ and include explicit expressions for the pseudoenergy and pseudomomentum. In $\S 4$ we show that the results of $\S 3$ can be generalized to the case of a veering background flow. We highlight the relationship between pseudoenergy and pseudomomentum and discuss important applications to gravity-wave parameterization in $\S 5$. Finally, we conclude with a brief summary and discussion in $\S 6$.

\section{Three-dimensional anelastic and Boussinesq equations}

We consider the most general form of the anelastic approximation as derived by Lipps \& Hemler (1982). The system of equations is

$$
\begin{aligned}
\frac{\partial \boldsymbol{v}}{\partial t}+(\boldsymbol{v} \cdot \nabla) \boldsymbol{v} & =-\nabla\left(c_{p} \theta_{0} \tilde{\boldsymbol{\pi}}\right)-c_{p} \frac{\mathrm{d} \pi_{0}}{\mathrm{~d} z} \tilde{\theta} \hat{\boldsymbol{z}}, \\
\frac{\partial \tilde{\theta}}{\partial t}+(\boldsymbol{v} \cdot \nabla) \tilde{\theta}+w \frac{\mathrm{d} \theta_{0}}{\mathrm{~d} z} & =0, \\
\nabla \cdot\left(\rho_{0} \boldsymbol{v}\right) & =0,
\end{aligned}
$$


where $\boldsymbol{v}$ is the three-dimensional velocity vector (with $w$ as its vertical component) and the potential temperature $\theta=T\left(p / p_{00}\right)^{-\kappa}$ and Exner function $\pi=\left(p / p_{00}\right)^{\kappa}$ have been decomposed as

$$
\begin{aligned}
& \theta=\theta_{0}(z)+\tilde{\theta}(x, y, z, t), \\
& \pi=\pi_{0}(z)+\tilde{\pi}(x, y, z, t),
\end{aligned}
$$

with $p_{00}$ as a reference pressure and $\kappa=R / c_{p}$, and $\rho_{0}(z)$ is the background density. The vertically dependent background state $\theta_{0}, \pi_{0}$ is in hydrostatic balance

$$
\frac{\mathrm{d} \pi_{0}}{\mathrm{~d} z}=-\frac{g}{c_{p} \theta_{0}} .
$$

As the Boussinesq equations are a subset of the anelastic system one can obtain the Boussinesq system from (2.1) by taking the limits

$$
\rho_{0} \rightarrow \rho_{*}, \quad \theta_{0} \rightarrow \theta_{*}, \quad \pi_{0} \rightarrow-\frac{g z}{c_{p} \theta_{*}}, \quad \theta \rightarrow-\frac{\theta_{*}}{\rho_{*}} \rho,
$$

where the star subscript refers to a constant background state. Here the second expression applies only where $\theta_{0}$ is not differentiated, while the fourth expression applies to $\tilde{\theta}$ and to $\theta_{0}$ when it is differentiated.

\subsection{Hamiltonian structure}

In order to apply Hamiltonian theory, the systems of equations must be energetically closed. As for the two-dimensional anelastic system, energy is conserved in the threedimensional anelastic system and the Hamiltonian is

$$
\mathscr{H}=\int\left(\frac{\rho_{0}}{2}|\boldsymbol{v}|^{2}+c_{p} \rho_{0} \pi_{0} \theta\right) \mathrm{d} V
$$

(Scinocca \& Shepherd 1992), where the integral is over the three-dimensional domain $\mathrm{d} V=\mathrm{d} x \mathrm{~d} y \mathrm{~d} z$. In the Boussinesq case the Hamiltonian is

$$
\mathscr{H}_{B}=\int\left(\frac{\rho_{*}}{2}|\boldsymbol{v}|^{2}+\rho g z\right) \mathrm{d} V .
$$

We also have, according to Noether's theorem, conservation of $x$ - and $y$-momentum due to invariance under translations in $x$ and $y$. The momentum functionals for the anelastic and Boussinesq systems are respectively

$$
\mathscr{M}=\int \rho_{0} \boldsymbol{u} \mathrm{d} V, \quad \mathscr{M}_{B}=\int \rho_{*} \boldsymbol{u} \mathrm{d} V
$$

(Scinocca \& Shepherd 1992), where $\boldsymbol{u}$ is the horizontal component of the velocity vector.

In the anelastic system both $\theta$ and the potential vorticity $q=\omega \cdot \nabla \theta / \rho_{0}$ are materially conserved, where $\omega=\nabla \times \boldsymbol{v}$, while for the Boussinesq system both $\rho$ and $q=\omega \cdot \nabla \rho / \rho_{*}$ are materially conserved. In each case these lead to Casimir invariants in the usual way (Shepherd 1990). In the case of the anelastic and Boussinesq systems we have, respectively,

$$
\mathscr{C}=\int \rho_{0} C(\theta, q) \mathrm{d} V \text { and } \mathscr{C}_{B}=\int \rho_{*} C(\rho, q) \mathrm{d} V .
$$

The functional form of the Casimir functions $C(\cdot, \cdot)$ is central to the derivation of wave activities. For convenience, we ignore possible boundary contributions to the wave activities. This still retains all the essential physics. 


\section{Wave-activity conservation laws for a plane-parallel background flow}

For each system we decompose all fields into a steady plane-parallel background flow (without loss of generality, we take the flow to be oriented in the $\hat{\boldsymbol{x}}$-direction) and deviations from that state (not necessarily small),

$$
\begin{aligned}
\boldsymbol{v} & =U(z) \hat{\boldsymbol{x}}+\boldsymbol{v}^{\prime}(x, y, z, t), \\
\theta & =\Theta(z)+\theta^{\prime}(x, y, z, t),
\end{aligned}
$$

where $\theta_{0}(z)$ has been absorbed into $\Theta(z)$. Such a background state has been used in many studies of gravity-wave propagation (Eliassen \& Palm 1961; Bretherton 1966; Hines \& Reddy 1967). Abarbanel et al. (1986) noted the degeneracy of such a plane-parallel background flow in the context of their Hamiltonian nonlinear stability analysis of stratified fluid equilibria - without a background potential vorticity gradient one cannot construct a pseudoenergy or pseudomomentum in the usual way. Following their analysis we introduce a small slowly varying $y$ dependence to the plane-parallel flow to break the degeneracy. Thus

$$
\boldsymbol{v}=V(y, z) \hat{\boldsymbol{x}}+\boldsymbol{v}^{\prime}(x, y, z, t)
$$

where

$$
\begin{aligned}
V(y, z) & =U(z)+\gamma(y), \\
\gamma(y) & =\gamma_{0}\left(\frac{y^{2}}{2 L^{2}}\right), \quad \gamma_{0} \ll U(z),
\end{aligned}
$$

with $\gamma_{0}$ a reference velocity scale and $|y| \ll L$. The length scale $L$ is large compared to any other length scale in the problem. All quantities are expanded in $y / L$ and we require the leading-order contributions to the wave activities to be independent of $\gamma_{0}$ (i.e. they should not vanish as $\gamma_{0} \rightarrow 0$ ). Essentially, $(3.3 b)$ introduces a limiting background potential vorticity gradient

$$
Q(y, z)=-\frac{\gamma_{0} y}{\rho_{0} L^{2}} \Theta_{z}
$$

which encodes some Lagrangian information and removes the degeneracy. Upon deriving the wave activities we take the limit $\gamma_{0} \rightarrow 0$ and, correspondingly, set the disturbance potential vorticity $q^{\prime}=q-Q$ equal to zero. In a plane-parallel background flow with zero potential vorticity, a non-zero $q^{\prime}$ could only arise from diabatic processes.

\subsection{Pseudoenergy}

The pseudoenergy functional is defined by

$$
\mathscr{A}^{\mathscr{E}}=\mathscr{H}(\xi)+\mathscr{C}^{\mathscr{E}}(\xi)-\mathscr{H}(X)-\mathscr{C}^{\mathscr{E}}(X)
$$

where $\xi$ is the state vector (a vector of the dependent variables), $X$ is the background state, subject to the condition that the first variation of $\mathscr{A}^{\mathscr{E}}$ vanish when $\xi=X$, and the Casimir $\mathscr{C}^{\mathscr{E}}$ is defined by

$$
\left.\frac{\delta \mathscr{H}}{\delta \xi}\right|_{\xi=X}=-\left.\frac{\delta \mathscr{C}^{\mathscr{E}}}{\delta \xi}\right|_{\xi=X}
$$

where $\delta / \delta \xi$ represents the usual functional derivative. This construction ensures that the pseudoenergy is both quadratic in disturbance amplitude and conserved - in 
contrast to both the wave energy, which is quadratic but not conserved, and the disturbance energy, which is conserved but not quadratic. Taking $\xi=(\boldsymbol{v}, \theta)$, we have

$$
\begin{aligned}
& \frac{\delta \mathscr{H}}{\delta \boldsymbol{v}}=\rho_{0} \boldsymbol{v}, \quad \frac{\delta \mathscr{H}}{\delta \theta}=c_{p} \rho_{0} \pi_{0}, \\
& \frac{\delta \mathscr{C}^{\mathscr{E}}}{\delta \boldsymbol{v}}=C_{q q}^{\mathscr{E}} \nabla q \times \nabla \theta, \quad \frac{\delta \mathscr{C}^{\mathscr{E}}}{\delta \theta}=\rho_{0} C_{\theta}^{\mathscr{E}}-\nabla C_{q}^{\mathscr{E}} \cdot(\nabla \times \boldsymbol{v}) .
\end{aligned}
$$

Thus, in order to satisfy the extremal conditions (3.6) the pseudoenergy Casimir density must satisfy

$$
\begin{aligned}
\rho_{0} V \hat{\boldsymbol{x}} & =C_{q q}^{\mathscr{E}} \nabla \Theta \times \nabla Q, \\
\rho_{0} C_{\theta}^{\mathscr{E}} & =-c_{p} \rho_{0} \pi_{0}+\nabla C_{q}^{\mathscr{E}} \cdot(\nabla \times V \hat{\boldsymbol{x}})
\end{aligned}
$$

where subscripts $q$ and $\theta$ denote partial derivatives (always taken to be evaluated at the background state $(Q, \Theta))$, which imply to leading order in $O(y / L)$ (see the Appendix)

$$
\begin{aligned}
C_{q q}^{\mathscr{E}} & =-\frac{\rho_{0} U}{Q_{y} \Theta_{z}}=\frac{\rho_{0}^{2} U L^{2}}{\gamma_{0}\left(\Theta_{z}\right)^{2}}, \\
C_{\theta \theta}^{\mathscr{E}} & =\frac{g}{\theta_{0} \Theta_{z}}-\frac{1}{\Theta_{z}}\left(\frac{\left(U^{2}\right)_{z}}{2 \Theta_{z}}\right)_{z}, \\
C_{\theta q}^{\mathscr{E}} & =\frac{\gamma_{y}}{\gamma_{y y} \Theta_{z}}\left[\frac{\rho_{0} U_{z}}{\Theta_{z}}-2\left(\frac{\rho_{0} U}{\Theta_{z}}\right)_{z}\right] .
\end{aligned}
$$

The right-hand sides of (3.9) are to be regarded as functions of $(Q, \Theta)$, via their dependence on $(y, z)$. In order for $y(Q, \Theta)$ and $z(Q, \Theta)$ to be well-defined, the transformation $(y, z) \rightarrow(Q, \Theta)$ must be invertible. This requires

$$
\frac{\partial(Q, \Theta)}{\partial(y, z)}=-\frac{\gamma_{0}\left(\Theta_{z}\right)^{2}}{\rho_{0} L^{2}} \neq 0
$$

where $\partial(\cdot, \cdot) / \partial(\cdot, \cdot)$ is the Jacobian. The same is required of the Boussinesq background state $(Q, R)$, where $R$ is the background density.

The general quadratic form of the anelastic pseudoenergy density is

$$
A^{\mathscr{E}}=\frac{\rho_{0}}{2}\left[|\delta \boldsymbol{v}|^{2}+C_{\theta \theta}^{\mathscr{E}}(\delta \theta)^{2}+C_{q}^{\mathscr{E}} \delta^{2} q\right]
$$

where terms involving $\delta q$ have been set equal to zero as described previously. The last term can be decomposed as

$$
\frac{\rho_{0}}{2} C_{q}^{\mathscr{E}} \delta^{2} q=\frac{\rho_{0} U}{\Theta_{z}} \delta \theta \delta \omega_{(y)}+y\left(\frac{\rho_{0} U}{\Theta_{z}}\right)_{z} \delta \theta \delta \omega_{(z)}
$$

and, using $\delta q=0$, we have $\delta \omega_{(z)}=-U_{z} \delta \theta_{y} / \Theta_{z}$ which implies

$$
\begin{aligned}
\frac{\rho_{0}}{2} C_{q}^{\mathscr{E}} \delta^{2} q & =\frac{\rho_{0} U}{\Theta_{z}} \delta \theta \delta \omega_{(y)}-y \frac{U_{z}}{\Theta_{z}}\left(\frac{\rho_{0} U}{\Theta_{z}}\right)_{z}\left(\frac{1}{2}(\delta \theta)^{2}\right)_{y} \\
& =\frac{\rho_{0} U}{\Theta_{z}} \delta \theta \delta \omega_{(y)}+\frac{U_{z}}{\Theta_{z}}\left(\frac{\rho_{0} U}{\Theta_{z}}\right)_{z} \frac{1}{2}(\delta \theta)^{2},
\end{aligned}
$$

the last step following from integration by parts. We note that only the $\delta \omega_{(z)}$ term can be re-written in terms of $\delta \theta$, as there is no constraint placed on $\delta \omega_{(y)}$ from $\delta q=0$. 
Thus, the quadratic pseudoenergy for the anelastic system is

$$
\mathscr{A}^{\mathscr{E}}=\int\left[\frac{\rho_{0}}{2}\left|\boldsymbol{v}^{\prime}\right|^{2}+\frac{\rho_{0}}{2}\left[\frac{g^{2}}{\theta_{0}^{2} N^{2}}-\frac{\rho_{0} U}{\left(\Theta_{z}\right)^{2}}\left(\frac{U_{z}}{\rho_{0}}\right)_{z}\right]\left(\theta^{\prime}\right)^{2}+\frac{\rho_{0} U}{\Theta_{z}} \omega_{(y)}^{\prime} \theta^{\prime}\right] \mathrm{d} V,
$$

where $N^{2}=g \Theta_{z} / \theta_{0}$. In the Boussinesq case we obtain

$$
\mathscr{A}_{B}^{\mathscr{E}}=\int\left[\frac{\rho_{*}}{2}\left|\boldsymbol{v}^{\prime}\right|^{2}+\frac{\rho_{*}}{2}\left[\frac{g^{2}}{\rho_{*}^{2} N^{2}}-\frac{U}{\left(R_{z}\right)^{2}} U_{z z}\right]\left(\rho^{\prime}\right)^{2}+\frac{\rho_{*} U}{R_{z}} \omega_{(y)}^{\prime} \rho^{\prime}\right] \mathrm{d} V,
$$

where $N^{2}=-g R_{z} / \rho_{*}$. The pseudoenergy densities are simply the integrands of (3.14) and (3.15). We note that for either system, in the case of two-dimensional disturbances, which have no $y$-dependence, we recover (5.20b) and (5.21b) of Scinocca \& Shepherd (1992). We note that the gravity-wave energy considered by earlier authors (Eliassen \& Palm 1961, etc.) includes only the first two terms of these expressions.

Next we must show that (3.14) and (3.15) are conserved by the linearized dynamics with $V=U(z)$. Upon taking time derivatives we obtain

$$
\begin{aligned}
\frac{\partial A^{\mathscr{\delta}}}{\partial t}= & -\frac{\partial}{\partial x}\left[\frac{\rho_{0} U}{2}\left(u^{\prime}\right)^{2}+\frac{\rho_{0} U^{2}}{\Theta_{z}} \theta^{\prime} \omega_{(y)}^{\prime}-\frac{1}{2} \frac{\rho_{0}^{2} U^{2}}{\left(\Theta_{z}\right)^{2}}\left(\frac{U_{z}}{\rho_{0}}\right)_{z}\left(\theta^{\prime}\right)^{2}\right] \\
& -\frac{\partial}{\partial x}\left[c_{p} \rho_{0} \theta_{0} \pi^{\prime} u^{\prime}+\frac{\rho_{0} g U}{2 \theta_{0} \Theta_{z}}\left(\theta^{\prime}\right)^{2}\right] \\
& -\frac{\partial}{\partial y}\left[c_{p} \rho_{0} \theta_{0} \pi^{\prime} v^{\prime}+\rho_{0} U u^{\prime} v^{\prime}-\frac{\rho_{0}\left(U^{2}\right)_{z}}{2 \Theta_{z}} \theta^{\prime} v^{\prime}\right] \\
& -\frac{\partial}{\partial z}\left[c_{p} \rho_{0} \theta_{0} \pi^{\prime} w^{\prime}+\rho_{0} U u^{\prime} w^{\prime}\right] .
\end{aligned}
$$

In the Boussinesq case we obtain

$$
\begin{aligned}
\frac{\partial A_{B}^{\delta}}{\partial t}= & -\frac{\partial}{\partial x}\left[\frac{\rho_{*} U}{2}\left(u^{\prime}\right)^{2}+\frac{\rho_{*} U^{2}}{R_{z}} \rho^{\prime} \omega_{(y)}^{\prime}-\frac{1}{2} \frac{\rho_{*} U^{2}}{\left(R_{z}\right)^{2}} U_{z z}\left(\rho^{\prime}\right)^{2}\right] \\
& -\frac{\partial}{\partial x}\left[p^{\prime} u^{\prime}-\frac{g U}{2 R_{z}}\left(\rho^{\prime}\right)^{2}\right] \\
& -\frac{\partial}{\partial y}\left[p^{\prime} v^{\prime}+\rho_{*} U u^{\prime} v^{\prime}-\frac{\rho_{*}\left(U^{2}\right)_{z}}{2 R_{z}} \rho^{\prime} v^{\prime}\right] \\
& -\frac{\partial}{\partial z}\left[p^{\prime} w^{\prime}+\rho_{*} U u^{\prime} w^{\prime}\right] .
\end{aligned}
$$

Clearly both (3.16) and (3.17) can be written in the form (1.1) with an appropriately defined $\boldsymbol{F}$, and are hence conserved. The vertical fluxes are equal to those derived by Hines \& Reddy (1967) under simplifying assumptions (ignoring $U_{z}$ and $U_{z z}$ ). The vertical flux differs from that derived by Scinocca \& Shepherd (1992) in the two-dimensional case, which is $\psi^{\prime} \partial_{z} \psi_{t}^{\prime}$; our form shows explicitly the role of the pressure-work term $\pi^{\prime} w^{\prime}$ (Hines \& Reddy 1967). In the appropriate WKB limit both the densities and corresponding fluxes have slowly varying coefficients and hence satisfy the requirements of the group-velocity property (Vanneste \& Shepherd 1998). 


\subsection{Pseudomomentum}

The $x$-pseudomomentum functional is defined by

$$
\mathscr{A}^{\mathscr{P}_{x}}=\mathscr{M}(\xi)+\mathscr{C}^{\mathscr{P}_{x}}(\xi)-\mathscr{M}(X)-\mathscr{C}^{\mathscr{P}_{x}}(X)
$$

where the Casimir $\mathscr{C}^{\mathscr{P}_{x}}$ must satisfy

$$
\left.\frac{\delta \mathscr{M}}{\delta \xi}\right|_{\xi=X}=-\left.\frac{\delta \mathscr{C}^{\mathscr{P}_{x}}}{\delta \xi}\right|_{\xi=X}
$$

to ensure the background state is an extremal. Again taking $\xi=(\boldsymbol{v}, \theta)$, we have

$$
\begin{aligned}
\frac{\delta \mathscr{M}}{\delta \boldsymbol{v}} & =\rho_{0} \hat{\boldsymbol{x}}, \quad \frac{\delta \mathscr{M}}{\delta \theta}=0, \\
\frac{\delta \mathscr{C}^{\mathscr{P}_{x}}}{\delta \boldsymbol{v}} & =C_{q q}^{\mathscr{P}_{x}} \nabla q \times \nabla \theta, \quad \frac{\delta \mathscr{C}^{\mathscr{P}_{x}}}{\delta \theta}=\rho_{0} C_{\theta}^{\mathscr{P}_{x}}-\nabla C_{q}^{\mathscr{P}_{x}} \cdot(\nabla \times \boldsymbol{v}) .
\end{aligned}
$$

The pseudomomentum Casimir density must satisfy

$$
\begin{aligned}
\rho_{0} \hat{\boldsymbol{x}} & =C_{q q}^{\mathscr{P}_{x}} \nabla \Theta \times \nabla Q, \\
\rho_{0} C_{\theta}^{\mathscr{P}_{x}} & =\nabla C_{q}^{\mathscr{P}_{x}} \cdot(\nabla \times V \hat{\boldsymbol{x}})
\end{aligned}
$$

which imply to leading order (see the Appendix)

$$
\begin{aligned}
C_{q q}^{\mathscr{P}_{x}} & =-\frac{\rho_{0}}{Q_{y} \Theta_{z}}=\frac{\rho_{0}^{2} L^{2}}{\gamma_{0}\left(\Theta_{z}\right)^{2}}, \\
C_{\theta \theta}^{\mathscr{P}_{x}} & =-\frac{1}{\Theta_{z}}\left(\frac{U_{z}}{\Theta_{z}}\right)_{z}, \\
C_{\theta q}^{\mathscr{P}_{x}} & =-\frac{2 \gamma_{y}}{\gamma_{y y} \Theta_{z}}\left(\frac{\rho_{0}}{\Theta_{z}}\right)_{z} .
\end{aligned}
$$

Proceeding as in the case of the pseudoenergy, the quadratic pseudomomentum for the anelastic system is

$$
\mathscr{A}^{\mathscr{P}_{x}}=\int\left[-\frac{1}{2} \frac{\rho_{0}^{2}}{\left(\Theta_{z}\right)^{2}}\left(\frac{U_{z}}{\rho_{0}}\right)_{z}\left(\theta^{\prime}\right)^{2}+\frac{\rho_{0}}{\Theta_{z}} \omega_{(y)}^{\prime} \theta^{\prime}\right] \mathrm{d} V .
$$

In the Boussinesq case we obtain

$$
\mathscr{A}_{B}^{\mathscr{P}_{x}}=\int\left[-\frac{1}{2} \frac{\rho_{*}}{\left(R_{z}\right)^{2}} U_{z z}\left(\rho^{\prime}\right)^{2}+\frac{\rho_{*}}{R_{z}} \omega_{(y)}^{\prime} \rho^{\prime}\right] \mathrm{d} V .
$$

We note again that for either system, in the limiting case of two-dimensional disturbances, we recover (6.15) and (6.16) of Scinocca \& Shepherd (1992). We note that in the earlier three-dimensional studies discussed previously, while there is a discussion of momentum flux, they do not quantify wave momentum (i.e. pseudomomentum) as has been done here.

Next we must show that (3.23) and (3.24) are conserved by the linearized dynamics with $V=U(z)$. Upon taking time derivatives we obtain

$$
\begin{aligned}
\frac{\partial A^{\mathscr{P}_{x}}}{\partial t}= & -\frac{\partial}{\partial x}\left[\frac{\rho_{0}}{2}\left[\left(u^{\prime}\right)^{2}-\left(v^{\prime}\right)^{2}-\left(w^{\prime}\right)^{2}\right]+\frac{\rho_{0} U}{\Theta_{z}} \theta^{\prime} \omega_{(y)}^{\prime}-\frac{1}{2} \frac{\rho_{0}^{2} U}{\left(\Theta_{z}\right)^{2}}\left(\frac{U_{z}}{\rho_{0}}\right)_{z}\left(\theta^{\prime}\right)^{2}\right] \\
& -\frac{\partial}{\partial x}\left[\frac{\rho_{0} g}{2 \theta_{0} \Theta_{z}}\left(\theta^{\prime}\right)^{2}\right]-\frac{\partial}{\partial y}\left[\rho_{0} u^{\prime} v^{\prime}-\frac{\rho_{0} U_{z}}{\Theta_{z}} \theta^{\prime} v^{\prime}\right]-\frac{\partial}{\partial z}\left[\rho_{0} u^{\prime} w^{\prime}\right] .
\end{aligned}
$$


In the Boussinesq case we obtain

$$
\begin{aligned}
\frac{\partial A_{B}^{\mathscr{P}_{x}}}{\partial t}= & -\frac{\partial}{\partial x}\left[\frac{\rho_{*}}{2}\left[\left(u^{\prime}\right)^{2}-\left(v^{\prime}\right)^{2}-\left(w^{\prime}\right)^{2}\right]+\frac{\rho_{*} U}{R_{z}} \rho^{\prime} \omega_{(y)}^{\prime}-\frac{1}{2} \frac{\rho_{*} U}{\left(R_{z}\right)^{2}} U_{z z}\left(\rho^{\prime}\right)^{2}\right] \\
& +\frac{\partial}{\partial x}\left[\frac{g}{2 R_{z}}\left(\rho^{\prime}\right)^{2}\right]-\frac{\partial}{\partial y}\left[\rho_{*} u^{\prime} v^{\prime}-\frac{\rho_{*} U_{z}}{R_{z}} \rho^{\prime} v^{\prime}\right]-\frac{\partial}{\partial z}\left[\rho_{*} u^{\prime} w^{\prime}\right] .
\end{aligned}
$$

Once again both (3.25) and (3.26) can be written as (1.1), with an appropriate $\boldsymbol{F}$, and are hence conserved. As for the pseudoenergy, the vertical flux of pseudomomentum is equal to that derived by Hines \& Reddy (1967) under simplifying assumptions, and in the WKB limit the densities and corresponding fluxes have slowly varying coefficients and hence satisfy the requirements of the group-velocity property (Vanneste \& Shepherd 1998).

\section{Extension to a veering background flow}

An important extension of the results of $\S 3$ is to the case of non-plane-parallel flows, i.e. flows which veer with height. As before, we introduce a small slowly-varying crossstream dependence to the prevailing flow, except the latter is written in streamwise coordinates which can veer with height:

$$
\boldsymbol{v}=W \hat{\boldsymbol{s}}+\boldsymbol{v}^{\prime}(x, y, z, t)
$$

where

$$
W \hat{\boldsymbol{s}}=U(z) \hat{\boldsymbol{x}}+V(z) \hat{\boldsymbol{y}}+\gamma(n) \hat{\boldsymbol{s}} \quad \text { with } \quad \gamma \ll U(z), \quad V(z)
$$

where $n$ is the coordinate normal to the streamwise direction $\hat{s}$. The veering of the background flow is measured by the angle $\phi(z)$ between the background velocity vector and the $\hat{\boldsymbol{x}}$-direction. As in $\S 3$ we consider all wave activities in the limit of vanishing $\gamma_{0}$ and $q^{\prime}$.

Proceeding as in $\S 3$, the quadratic pseudoenergy is

$$
\begin{aligned}
\mathscr{A}^{\mathscr{E}}=\int\left[\frac{\rho_{0}}{2}\left|\boldsymbol{v}^{\prime}\right|^{2}+\frac{\rho_{0}}{2}\left\{\frac{g^{2}}{\theta_{0}^{2} N^{2}}-\frac{\rho_{0} U}{\left(\Theta_{z}\right)^{2}}(\right.\right. & \left.\left.\frac{U_{z}}{\rho_{0}}\right)_{z}-\frac{\rho_{0} V}{\left(\Theta_{z}\right)^{2}}\left(\frac{V_{z}}{\rho_{0}}\right)_{z}\right\}\left(\theta^{\prime}\right)^{2} \\
& \left.+\frac{\rho_{0} U}{\Theta_{z}} \omega_{(y)}^{\prime} \theta^{\prime}-\frac{\rho_{0} V}{\Theta_{z}} \omega_{(x)}^{\prime} \theta^{\prime}\right] \mathrm{d} V .
\end{aligned}
$$

Upon taking the time derivative of the pseudoenergy density and horizontally averaging we obtain

$$
\frac{\partial \bar{A}^{\mathscr{E}}}{\partial t}=-\frac{\partial}{\partial z}\left[c_{p} \rho_{0} \theta_{0} \overline{\pi^{\prime} w^{\prime}}+\rho_{0} U \overline{u^{\prime} w^{\prime}}+\rho_{0} V \overline{v^{\prime} w^{\prime}}\right]
$$

in the limit of slow veering, i.e. $\phi_{z} \ll U_{z} / U, V_{z} / V$, where the right-hand side is the usual vertical energy flux convergence.

In the case of the pseudomomentum we define streamwise $\mathscr{A}^{\mathscr{P}_{s}}$ and normal $\mathscr{A}^{\mathscr{P}_{n}}$ quadratic pseudomomentum components as

$$
\begin{array}{r}
\mathscr{A}^{\mathscr{P}_{s}}=\int\left[-\frac{1}{2} \frac{\rho_{0}^{2}}{\left(\Theta_{z}\right)^{2}}\left\{\cos \phi\left(\frac{U_{z}}{\rho_{0}}\right)_{z}+\sin \phi\left(\frac{V_{z}}{\rho_{0}}\right)_{z}\right\}\left(\theta^{\prime}\right)^{2}+\cos \phi \frac{\rho_{0}}{\Theta_{z}} \omega_{(y)}^{\prime} \theta^{\prime}\right. \\
\left.-\sin \phi \frac{\rho_{0}}{\Theta_{z}} \omega_{(x)}^{\prime} \theta^{\prime}\right] \mathrm{d} V
\end{array}
$$




$$
\begin{array}{r}
\mathscr{A}^{\mathscr{P}_{n}}=\int\left[-\frac{1}{2} \frac{\rho_{0}^{2}}{\left(\Theta_{z}\right)^{2}}\left\{\sin \phi\left(\frac{U_{z}}{\rho_{0}}\right)_{z}-\cos \phi\left(\frac{V_{z}}{\rho_{0}}\right)_{z}\right\}\left(\theta^{\prime}\right)^{2}+\sin \phi \frac{\rho_{0}}{\Theta_{z}} \omega_{(y)}^{\prime} \theta^{\prime}\right. \\
\left.+\cos \phi \frac{\rho_{0}}{\Theta_{z}} \omega_{(x)}^{\prime} \theta^{\prime}\right] \mathrm{d} V
\end{array}
$$

whose associated horizontally averaged wave-activity conservation laws can be combined to give the usual vertical fluxes of horizontal momentum

$$
\begin{gathered}
\frac{\partial}{\partial t}\left[\cos \phi \bar{A}^{\mathscr{P}_{s}}+\sin \phi \bar{A}^{\mathscr{P}_{n}}\right]=\frac{\partial \bar{A}^{\mathscr{P}_{x}}}{\partial t}=-\frac{\partial}{\partial z} \overline{\left[\rho_{0} u^{\prime} w^{\prime}\right]}, \\
\frac{\partial}{\partial t}\left[\sin \phi \bar{A}^{\mathscr{P}_{s}}-\cos \phi \bar{A}^{\mathscr{P}_{n}}\right]=\frac{\partial \bar{A}^{\mathscr{P}_{y}}}{\partial t}=-\frac{\partial}{\partial z} \overline{\left[\rho_{0} v^{\prime} w^{\prime}\right]},
\end{gathered}
$$

where $A^{\mathscr{P}_{x}}$ is given by (3.23) and $A^{\mathscr{P}_{y}}$ is the obvious analogue to it. We note that in the limit $\phi \rightarrow 0$, which corresponds to $V \rightarrow 0$, we recover the results of $\S 3$. Interestingly, under these limits the wave-activity conservation law for the normal component of pseudomomentum is directly related to the $y$ pseudomomentum flux. Yet the $y$ pseudomomentum cannot be defined for the plane-parallel background flow because there is no extremal condition.

\section{Noether's theorem and implications for gravity-wave parameterization}

There exists a general relationship between pseudoenergy and pseudomomentum, derivable from Noether's theorem, which is

$$
\mathscr{A}^{\mathscr{E}}=c \mathscr{A}^{\mathscr{P}}
$$

where $c$ is horizontal phase speed in the direction of the symmetry associated with $\mathscr{A}^{\mathscr{P}}$. The most concise derivation exploits the fact that using Noether's theorem the pseudoenergy and $x$-pseudomomentum are related to time and space symmetries according to

$$
\xi_{t}=J \frac{\delta \mathscr{A}^{\mathscr{E}}}{\delta \xi}, \quad-\xi_{x}=J \frac{\delta \mathscr{A}^{\mathscr{P}_{x}}}{\delta \xi}
$$

(e.g. Shepherd 1990), where $J$ is the symplectic operator in the Hamiltonian description, evaluated at the background state. For a disturbance propagating in the $\hat{\boldsymbol{x}}$-direction with a phase speed $c$, one has $\xi_{t}+c \xi_{x}=0$ and hence

$$
J \frac{\delta}{\delta \xi}\left(\mathscr{A}^{\mathscr{E}}-c \mathscr{A}^{\mathscr{P}_{x}}\right)=0
$$

which gives (5.1) assuming a non-singular $J$. Since this is the case for the linearized equations, the result (5.1) is only established here for the quadratic wave activities.

The relationship (5.1) can be shown explicitly to hold for the wave-activity densities derived in $\S 3$. In particular, it can be shown (after some algebra) that the integrands in (3.23) and (3.24) are equal to $k E / \hat{\sigma}$ where $E$ is the wave energy $\left(E=\rho_{0}\left(u^{\prime 2}+\right.\right.$ $\left.v^{\prime 2}+w^{\prime 2}\right) / 2+\rho_{0} g^{2} \theta^{\prime 2} / 2 N^{2} \theta_{0}^{2}$ for the anelastic case and $E=\rho_{*}\left(u^{\prime 2}+v^{\prime 2}+w^{\prime 2}\right) / 2+$ $\rho_{*} g^{2}\left(\rho^{\prime}\right)^{2} / 2 N^{2}\left(\rho_{*}\right)^{2}$ in the Boussinesq case), $k$ is the wavenumber in the $\hat{\boldsymbol{x}}$-direction, and $\hat{\sigma}=\sigma-U k$ is the intrinsic frequency which (under WKB conditions, but not neglecting the flow curvature $U_{z z}$ ) obeys the dispersion relation

$$
\hat{\sigma}^{2}\left(k^{2}+\ell^{2}+m^{2}\right)-N^{2}\left(k^{2}+\ell^{2}\right)+\hat{\sigma} k U_{z z}=0
$$


where $\ell$ and $m$ are the $y$ and $z$ wavenumbers, respectively. This is the usual relation involving the wave action $E / \hat{\sigma}$ (Andrews \& McIntyre 1978). Using also that the integrands in (3.14) and (3.15) equal $\sigma E / \hat{\sigma}$ we have that (5.1) holds for (3.14) and (3.23) as well as for (3.15) and (3.24), with $c=c_{x}=\sigma / k$.

Relation (5.1) also holds for the horizontally averaged, vertical components of the quadratic pseudoenergy and pseudomomentum fluxes, i.e. $\bar{F}_{(z)}^{\mathscr{E}}=c_{x} \bar{F}_{(z)}^{\mathscr{P}_{x}}$. This follows directly from (5.1) together with (1.1), assuming both fluxes vanish for some value of $z$, and takes the explicit form

$$
c_{p} \rho_{0} \theta_{0} \overline{\pi^{\prime} w^{\prime}}+\left(U-c_{x}\right) \rho_{0} \overline{u^{\prime} w^{\prime}}=0
$$

for the anelastic case and

$$
\overline{p^{\prime} w^{\prime}}+\left(U-c_{x}\right) \rho_{*} \overline{u^{\prime} w^{\prime}}=0
$$

for the Boussinesq case. This relationship between the vertical pseudoenergy and pseudomomentum fluxes was found by Eliassen \& Palm (1961), without using the Hamiltonian formalism, and is generally referred to as the first Eliassen-Palm theorem (Lindzen 1990). However that derivation assumes WKB conditions and furthermore neglects the $U_{z}$ and $U_{z z}$ terms, reducing waves in a stratified shear flow to Dopplershifted gravity waves. Our result is considerably more general.

For the veering background flow considered in $\S 4$, it can be shown that the integrands of (4.3), (4.5a), and (4.5b) are equal to $\sigma E / \hat{\sigma}, \cos \phi k E / \hat{\sigma}+\sin \phi \ell E / \hat{\sigma}$, and $\sin \phi k E / \hat{\sigma}-\cos \phi \ell E / \hat{\sigma}$, respectively. As a result, we obtain the following relationships between the vertical fluxes:

$$
\bar{F}_{(z)}^{\mathscr{E}}=c_{x} \bar{F}_{(z)}^{\mathscr{P}_{x}}=c_{y} \bar{F}_{(z)}^{\mathscr{P}_{y}} .
$$

Thus using the Hamiltonian formalism, we can further generalize the relationship between the vertical fluxes of pseudoenergy and pseudomomentum beyond the results of Eliassen \& Palm (1961), to the case of a veering background flow.

The relationship between the vertical pseudoenergy and pseudomomentum fluxes has interesting implications for gravity-wave parameterization. Most current gravitywave parameterizations account for the vertical flux of pseudomomentum, as is appropriate for a columnar approach, but do not usually account for the vertical flux of pseudoenergy in a consistent manner; one can then tune the gravity-wave momentum flux without a compensation in the energy flux (Becker 2004). It has been shown in observational and modelling studies that the energy flux and consequent turbulent dissipation due to gravity-wave breaking is non-negligible in the upper atmosphere (Lübken 1997; Becker 2004). The current analysis suggests that given a well-defined wave phase speed, one could determine the pseudoenergy flux from the pseudomomentum flux. In the same way that the pseudomomentum flux contributes to the mean momentum budget, the pseudoenergy flux contributes to the mean energy budget.

\section{Summary and discussion}

The anelastic and Boussinesq models play an important role in the numerical simulation and theoretical understanding of mesoscale phenomena. Here, wave-activity conservation laws for three-dimensional mesoscale disturbances to a horizontally homogeneous but vertically dependent background flow (both plane-parallel and veering) have been derived. These have direct application to the parameterization of subgrid-scale fluxes in climate models. The wave-activity fluxes provide a way of 
understanding subgrid-scale-mean-flow interactions, as the fluxes of pseudoenergy and pseudomomentum contribute to the mean energy and momentum budgets.

Scinocca \& Shepherd (1992) derived analogous wave-activity conservation laws for the two-dimensional anelastic and Boussinesq systems. Projecting the wave-activity densities (3.14), (3.15), (3.23), and (3.24) onto the $(x, z)$-plane we recover the results of Scinocca \& Shepherd (1992). Thus, the two-dimensional wave-activity conservation laws can be seen as limiting cases of the three-dimensional set-up. This is a non-trivial result because the two-dimensional analysis relied crucially on a special Casimir invariant which is particular to the two-dimensional geometry. When comparing the wave-activity densities in two and three dimensions it becomes clear that the limiting potential vorticity gradient is needed to generate the $\omega_{(y)}^{\prime} \theta^{\prime}$ term. The three-dimensional results derived here also make explicit the contribution of the pressure-work term to the vertical flux of pseudoenergy. They furthermore extend to the case of a veering background flow.

Wave activities for the three-dimensional Boussinesq equations have been considered long ago by Eliassen \& Palm (1961), Bretherton (1966), and Hines \& Reddy (1967). We have used the Hamiltonian framework of geophysical fluid dynamics to generalize and extend these earlier results in many ways: the results include a full dependence on the background flow (no WKB assumption) and require only the existence of horizontal and temporal homogeneity, they are extendable to finite amplitude, they can be generalized to the case of a veering wind, and wave activities were derived for both the three-dimensional anelastic and Boussinesq equations. The wave activities are derived using a limiting potential vorticity gradient, but the derived relations are independent of this gradient. Such a device was not needed in the earlier derivations which ignored $U_{z z}$. Thus, those authors were treating Doppler-shifted gravity waves, not waves in a stratified shear flow. In particular, their versions of (3.15) only contained the first two terms.

Relation (5.1) implies that the redistribution of energy in a background state accomplished by the generation, propagation, and dissipation of a wave with phase speed $c$, is equal to $c$ times the redistribution of momentum. This has important applications for gravity-wave parameterizations. In particular the connection (5.5) between the vertical fluxes of pseudoenergy and pseudomomentum, known for the plane-parallel background state under simplifying assumptions (Eliassen \& Palm 1961), has been here generalized using the Hamiltonian formalism.

Although the application of the current results to gravity-wave parameterization was highlighted, relationship (5.1) is completely general and applies to any phenomena modelled by the anelastic or Boussinesq equations, including convection and boundary-layer turbulence. Thus, the results apply directly to the parameterization of any subgrid-scale dynamical process.

This research has been supported by the Natural Sciences and Engineering Research Council of Canada, in part through a Canada Graduate Scholarship to the first author, and by the Canadian Foundation for Climate and Atmospheric Sciences.

\section{Appendix}

\section{A.1 Pseudoenergy Casimir derivatives}

Expressions (3.9a)-(3.9c) are derived as follows: from (3.8a)

$$
C_{q q}^{\mathscr{E}}=\frac{\rho_{0}^{2} L^{2}}{\gamma_{0}\left(\Theta_{z}\right)^{2}}\left[U+\frac{\gamma_{0} y^{2}}{2 L^{2}}\right],
$$


which reduces directly to $(3.9 a)$ to leading order. Then

$$
\begin{aligned}
C_{q q}^{\mathscr{E}} & =\frac{\partial\left(C_{q}^{\mathscr{E}}, \Theta\right)}{\partial(Q, \Theta)}=\frac{\partial\left(C_{q}^{\mathscr{E}}, \Theta\right)}{\partial(y, z)} \frac{\partial(y, z)}{\partial(Q, \Theta)}=-\left(C_{q}^{\mathscr{E}}\right)_{y} \Theta_{z} \frac{\rho_{0} L^{2}}{\gamma_{0}\left(\Theta_{z}\right)^{2}} \\
\Longleftrightarrow\left(C_{q}^{\mathscr{E}}\right)_{y} & =-\frac{\rho_{0}}{\Theta_{z}}\left[U+\frac{\gamma_{0} y^{2}}{2 L^{2}}\right] \Longrightarrow C_{q}^{\mathscr{E}}=-\frac{\rho_{0} U}{\Theta_{z}} y-\frac{\rho_{0} \gamma_{0}}{\Theta_{z} L^{2}} \frac{y^{3}}{6}
\end{aligned}
$$

which using (3.8b) implies

$$
\begin{aligned}
C_{\theta}^{\mathscr{E}} & =-c_{p} \pi_{0}+\frac{1}{\rho_{0}}\left[\left(C_{q}^{\mathscr{E}}\right)_{y} U_{z}-\left(C_{q}^{\mathscr{E}}\right)_{z} \frac{\gamma_{0} y}{L^{2}}\right] \\
& =-c_{p} \pi_{0}-\frac{U U_{z}}{\Theta_{z}}-\frac{\gamma_{0} y^{2}}{\rho_{0} L^{2}}\left[\frac{\rho_{0} U_{z}}{2 \Theta_{z}}-\left(\frac{\rho_{0} U}{\Theta_{z}}\right)_{z}\right]
\end{aligned}
$$

up to $O\left(y^{2} / L^{2}\right)$, and hence

$$
\begin{aligned}
C_{\theta \theta}^{\mathscr{E}}= & \frac{\partial\left(C_{\theta}^{\mathscr{E}}, Q\right)}{\partial(\Theta, Q)}=\frac{\partial\left(C_{\theta}^{\mathscr{E}}, Q\right)}{\partial(y, z)} \frac{\partial(y, z)}{\partial(\Theta, Q)}=-\frac{1}{\Theta_{z}}\left\{c_{p} \frac{\mathrm{d} \pi_{0}}{\mathrm{~d} z}+\left(\frac{\left(U^{2}\right)_{z}}{2 \Theta_{z}}\right)_{z}\right. \\
& \left.+\frac{\gamma_{0} y^{2}}{L^{2}}\left[\frac{1}{\rho_{0}}\left(\frac{\rho_{0} U}{\Theta_{z}}\right)_{z}\right]_{z}-\frac{\gamma_{0} y^{2}}{L^{2}}\left[\frac{U_{z}}{2 \Theta_{z}}\right]_{z}\right\}-\frac{\rho_{0} y}{\left(\Theta_{z}\right)^{2}}\left(\frac{\Theta_{z}}{\rho_{0}}\right)_{z}\left\{\frac { 2 \gamma _ { 0 } y } { \rho _ { 0 } L ^ { 2 } } \left[\frac{\rho_{0} U_{z}}{2 \Theta_{z}}\right.\right. \\
& \left.\left.-\left(\frac{\rho_{0} U}{\Theta_{z}}\right)_{z}\right]\right\}
\end{aligned}
$$

which reduces to $(3.9 b)$ in the appropriate limit. The final expression is derived using (A $2 b)$ and (A 3):

$$
\begin{aligned}
C_{\theta q}^{\mathscr{E}} & =\frac{\partial\left(C_{\theta}^{\mathscr{E}}, \Theta\right)}{\partial(Q, \Theta)}=\frac{\partial\left(C_{\theta}^{\mathscr{E}}, \Theta\right)}{\partial(y, z)} \frac{\partial(y, z)}{\partial(Q, \Theta)}=-\left(C_{\theta}^{\mathscr{E}}\right)_{y} \Theta_{z}\left(\frac{\rho_{0} L^{2}}{\gamma_{0}\left(\Theta_{z}\right)^{2}}\right) \\
& =\frac{\gamma_{y}}{\gamma_{y y} \Theta_{z}}\left[\frac{\rho_{0} U_{z}}{\Theta_{z}}-2\left(\frac{\rho_{0} U}{\Theta_{z}}\right)_{z}\right]
\end{aligned}
$$

and

$$
\begin{aligned}
C_{q \theta}^{\mathscr{E}} & =\frac{\partial\left(C_{q}^{\mathscr{E}}, Q\right)}{\partial(\Theta, Q)}=\frac{\partial\left(C_{q}^{\mathscr{E}}, Q\right)}{\partial(y, z)} \frac{\partial(y, z)}{\partial(\Theta, Q)} \\
& =\frac{\gamma_{y}}{\gamma_{y y} \Theta_{z}}\left\{-\left(\frac{\rho_{0} U}{\Theta_{z}}\right)_{z}+\frac{\rho_{0}^{2} U}{\left(\Theta_{z}\right)^{3}}\left(\frac{\Theta_{z}}{\rho_{0}}\right)_{z}+\frac{1}{2} \frac{\rho_{0} \gamma_{0} y^{2}}{\left(\Theta_{z}\right)^{3} L^{2}}\left(\frac{\Theta_{z}}{\rho_{0}}\right)_{z}\right\} \\
& =\frac{\gamma_{y}}{\gamma_{y y} \Theta_{z}}\left[\frac{\rho_{0} U_{z}}{\Theta_{z}}-2\left(\frac{\rho_{0} U}{\Theta_{z}}\right)_{z}\right] \\
& =C_{\theta q}^{\mathscr{E}}
\end{aligned}
$$

which are exactly $(3.9 c)$.

A.2 Pseudomomentum Casimir derivatives

Expressions (3.22a)-(3.22c) are derived as follows: from (3.21a)

$$
C_{q q}^{\mathscr{P}_{x}}=\frac{\rho_{0}^{2} L^{2}}{\gamma_{0}\left(\Theta_{z}\right)^{2}},
$$


which is exactly $(3.22 a)$. Using

$$
\begin{aligned}
& C_{q q}^{\mathscr{P}_{x}}=\frac{\partial\left(C_{q}^{\mathscr{P}_{x}}, \Theta\right)}{\partial(Q, \Theta)}=\frac{\partial\left(C_{q}^{\mathscr{P}_{x}}, \Theta\right)}{\partial(y, z)} \frac{\partial(y, z)}{\partial(Q, \Theta)}=-\left(C_{q}^{\mathscr{P}_{x}}\right)_{y} \Theta_{z} \frac{\rho_{0} L^{2}}{\gamma_{0}\left(\Theta_{z}\right)^{2}} \\
& \Longleftrightarrow\left(C_{q}^{\mathscr{P}_{x}}\right)_{y}=-\frac{\rho_{0}}{\Theta_{z}} \Longrightarrow C_{q}^{\mathscr{P}_{x}}=-\frac{\rho_{0} y}{\Theta_{z}}
\end{aligned}
$$

which using (3.21b) implies

$$
\begin{aligned}
C_{\theta}^{\mathscr{P}_{x}} & =\frac{1}{\rho_{0}}\left[\left(C_{q}^{\mathscr{P}_{x}}\right)_{y} U_{z}-\left(C_{q}^{\mathscr{P}_{x}}\right)_{z} \frac{\gamma_{0} y}{L^{2}}\right] \\
& =-\frac{U_{z}}{\Theta_{z}}+\left(\frac{\rho_{0}}{\Theta_{z}}\right)_{z} \frac{\gamma_{0} y^{2}}{\rho_{0} L^{2}}
\end{aligned}
$$

and hence

$$
\begin{aligned}
C_{\theta \theta}^{\mathscr{P}_{x}} & =\frac{\partial\left(C_{\theta}^{\mathscr{P}_{x}}, Q\right)}{\partial(\Theta, Q)}=\frac{\partial\left(C_{\theta}^{\mathscr{P}_{x}}, Q\right)}{\partial(y, z)} \frac{\partial(y, z)}{\partial(\Theta, Q)} \\
& =-\frac{1}{\Theta_{z}}\left\{\left(\frac{U_{z}}{\Theta_{z}}\right)_{z}-\frac{\gamma_{0} y^{2}}{L^{2}}\left[\frac{1}{\rho_{0}}\left(\frac{\rho_{0}}{\Theta_{z}}\right)_{z}\right]_{z}\right\}-\frac{2 \gamma_{0} y^{2}}{L^{2}\left(\Theta_{z}\right)^{2}}\left(\frac{\rho_{0}}{\Theta_{z}}\right)_{z}\left(\frac{\Theta_{z}}{\rho_{0}}\right)_{z}
\end{aligned}
$$

which reduces to $(3.22 b)$ to leading order. The final expression $(3.22 c)$ is derived using (A $7 b)$ and (A 8):

$$
\begin{aligned}
C_{\theta q}^{\mathscr{P}_{x}} & =\frac{\partial\left(C_{\theta}^{\mathscr{P}_{x}}, \Theta\right)}{\partial(Q, \Theta)}=\frac{\partial\left(C_{\theta}^{\mathscr{P}_{x}}, \Theta\right)}{\partial(y, z)} \frac{\partial(y, z)}{\partial(Q, \Theta)}=-\left(C_{\theta}^{\mathscr{P}_{x}}\right)_{y} \Theta_{z}\left(\frac{\rho_{0} L^{2}}{\gamma_{0}\left(\Theta_{z}\right)^{2}}\right) \\
& =-\frac{2 \gamma_{y}}{\gamma_{y y} \Theta_{z}}\left(\frac{\rho_{0}}{\Theta_{z}}\right)_{z}
\end{aligned}
$$

and

$$
\begin{aligned}
C_{q \theta}^{\mathscr{P}_{x}} & =\frac{\partial\left(C_{q}^{\mathscr{P}_{x}}, Q\right)}{\partial(\Theta, Q)}=\frac{\partial\left(C_{q}^{\mathscr{P}_{x}}, Q\right)}{\partial(y, z)} \frac{\partial(y, z)}{\partial(\Theta, Q)} \\
& =\frac{\gamma_{y}}{\gamma_{y y} \Theta_{z}}\left\{-\left(\frac{\rho_{0}}{\Theta_{z}}\right)_{z}+\frac{\rho_{0}^{2}}{\left(\Theta_{z}\right)^{2}}\left(\frac{\Theta_{z}}{\rho_{0}}\right)_{z}\right\} \\
& =-\frac{2 \gamma_{y}}{\gamma_{y y} \Theta_{z}}\left(\frac{\rho_{0}}{\Theta_{z}}\right)_{z} \\
& =C_{\theta q}^{\mathscr{P}_{x}}
\end{aligned}
$$

which is exactly $(3.22 c)$.

\section{REFERENCES}

Abarbanel, H. D. I., Holm, D. D., Marsden, J. E. \& Ratiu, T. S. 1986 Nonlinear stability analysis of stratified fluid equilibria. Phil. Trans. R. Soc. Lond. A 318, 349-409.

Andrews, D. G., Holton, J. R. \& Leovy, C. B. 1987 Middle Atmosphere Dynamics. Academic.

Andrews, D. G. \& McIntyre, M. E. 1978 On wave-action and its relatives. J. Fluid Mech. 89, 647-664.

BeCKer, E. 2004 Direct heating rates associated with gravity wave saturation. J. Atmos. Solar-Terres. Phys. 66, 683-696.

Bretherton, F. P. 1966 The propagation of groups of internal gravity waves in a shear flow. Q. J. R. Met. Soc. 92, 466-480. 
Eliassen, A. \& Palm, E. 1961 On the transfer of energy by mountain waves. Geofysiske Publ. 22, 94-101.

Hines, C. O. \& RedDy, C. A. 1967 On the propagation of atmospheric gravity waves through regions of wind shear. J. Geophys. Res. 72, 1015-1034.

Lindzen, R. S. 1990 Dynamics in Atmospheric Physics: Lecture Notes for an Introductory Graduatelevel Course. Cambridge University Press.

Lipps, F. B. \& Hemler, R. S. 1982 On the anelastic approximation for deep convection. J. Atmos. Sci. 39, 2192-2210.

LÜBKEN, F.-J. 1997 Seasonal variation of turbulent energy dissipation rates at high latitudes as determined by in situ measurements of neutral density fluctuations. J. Geophys. Res. 102, $13441-13456$.

SCINOCCA, J. F. \& SHEPHERd, T. G. 1992 Nonlinear wave-activity conservation laws and Hamiltonian structure for the two-dimensional anelastic equations. J. Atmos. Sci. 49, 5-27.

Shepherd, T. G. 1990 Symmetries, conservation laws, and Hamiltonian structure in geophysical fluid dynamics. Adv. Geophys. 32, 287-338.

Shepherd, T. G. 2003 Hamiltonian dynamics. In Encyclopedia of Atmospheric Sciences (ed. J. R. Holton et al.), pp. 929-938. Academic.

VANNESTE, J. \& SHEPHERD, T. G. 1998 On the group-velocity property for wave-activity conservation laws. J. Atmos. Sci. 55, 1063-1068. 